Supporting Information

\title{
Dynamics Transition of Polymer Films Induced by Polymer- Obstacle Entanglements on Rough Surfaces
}

\author{
Jiaxiang Li ${ }^{\mathrm{a}, \mathrm{b}}$, Yuyuan Lu ${ }^{\mathrm{a}}$, Lili Hao ${ }^{\mathrm{a}, \mathrm{b}}$, Ran Zhang ${ }^{\mathrm{a}}$, Mingming Ding ${ }^{\mathrm{a},{ }^{*}}$, \\ and Tongfei Shi ${ }^{\mathrm{a}, \mathrm{b}, *}$
}

${ }^{a}$ State Key Laboratory of Polymer Physics and Chemistry, Changchun Institute of Applied Chemistry, Chinese Academy of Sciences, Changchun 130022, P. R. China

${ }^{b}$ School of Applied Chemistry and Engineering, University of Science and Technology of China, Hefei 230026, P. R. China

${ }^{*}$ Corresponding author. E-mail: mmding@ciac.ac.cn; tfshi@ciac.ac.cn 


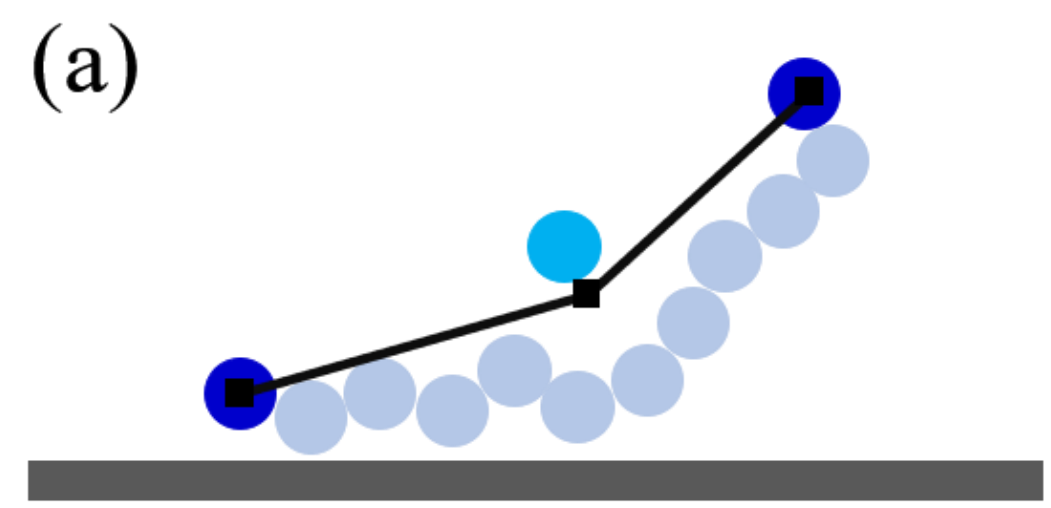

\section{(b)}

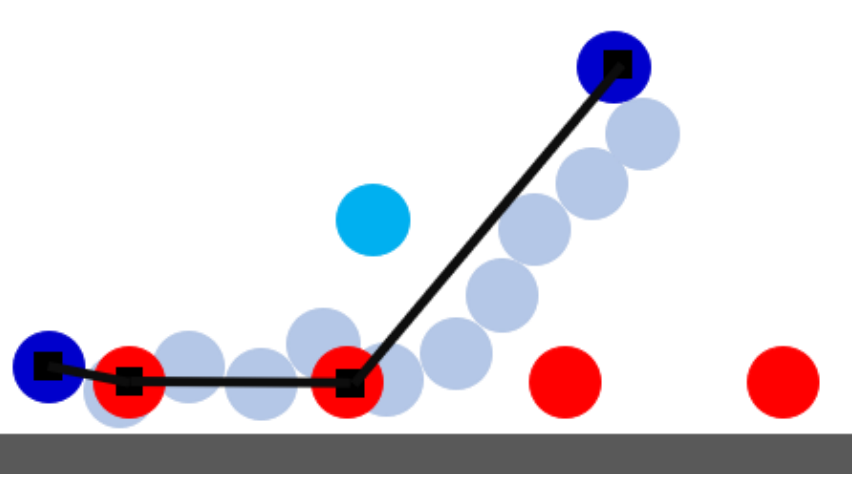

Figure S1: Schematic representation of (a) the shift of the primitive path near the smooth surface and (b) the anchored primitive path on the obstacles near the rough surfaces. 

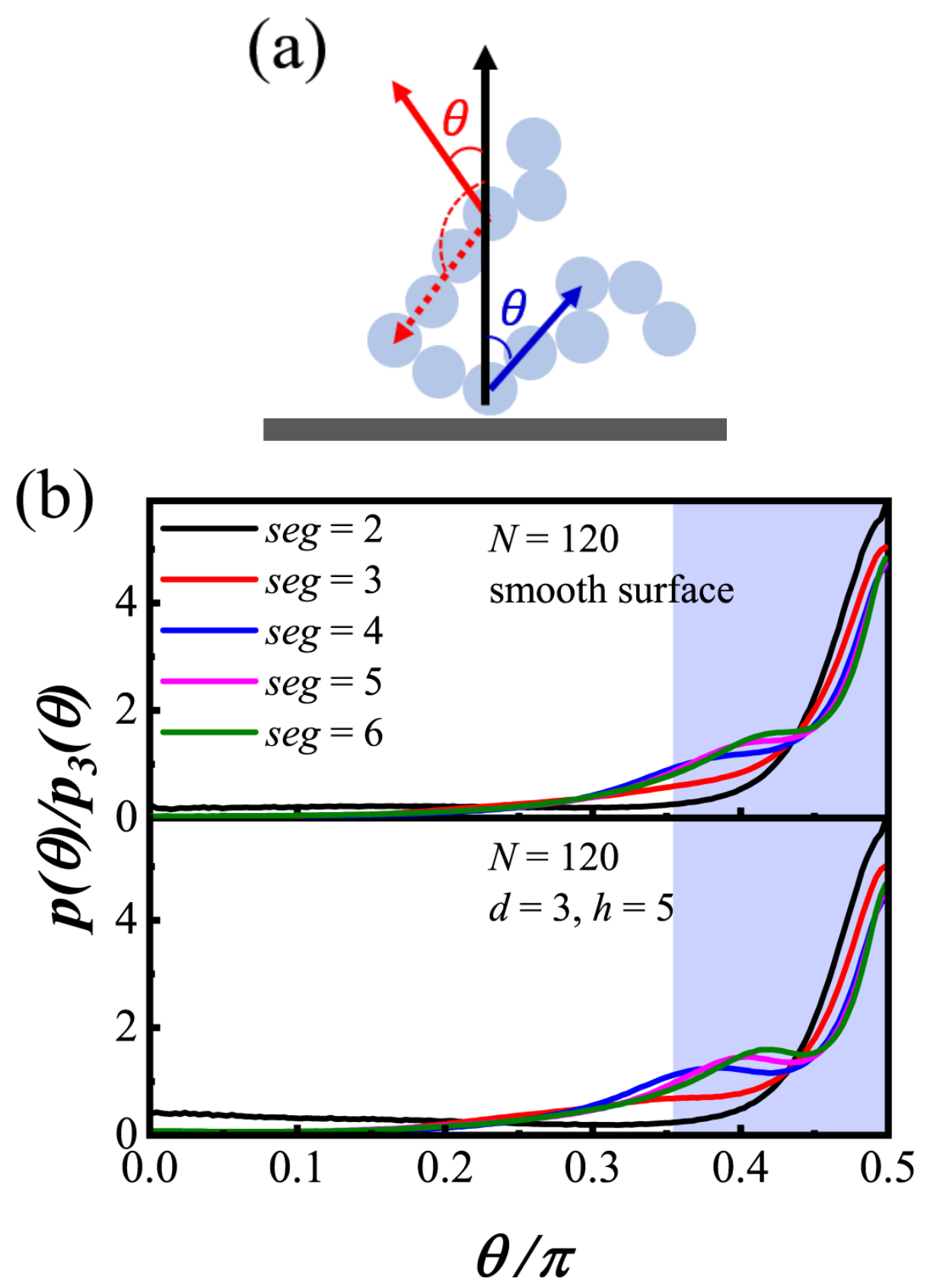

Figure S2: (a) Schematic representation of the definition of the orientation angle $\theta$. The blue arrow represents the angle calculated directly, and the red arrow represents the angle calculated after the symmetry transformation. (b) Orientation distribution $p(\theta)$ of the polymer segments with length seg $=2-6$ in the nearest layer $(z=1)$. 


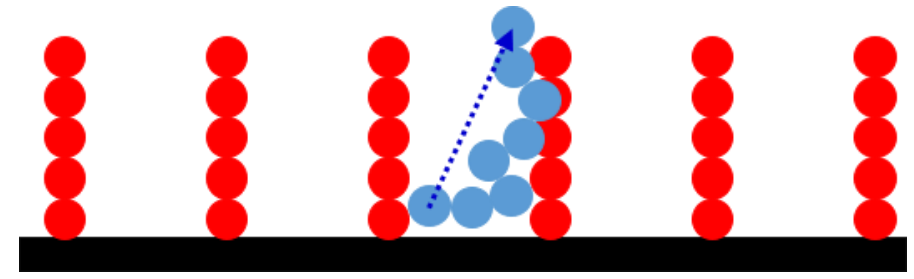

Figure S3: Schematic representation of the orientation tendency of the polymer segments in the array of the obstacles. 

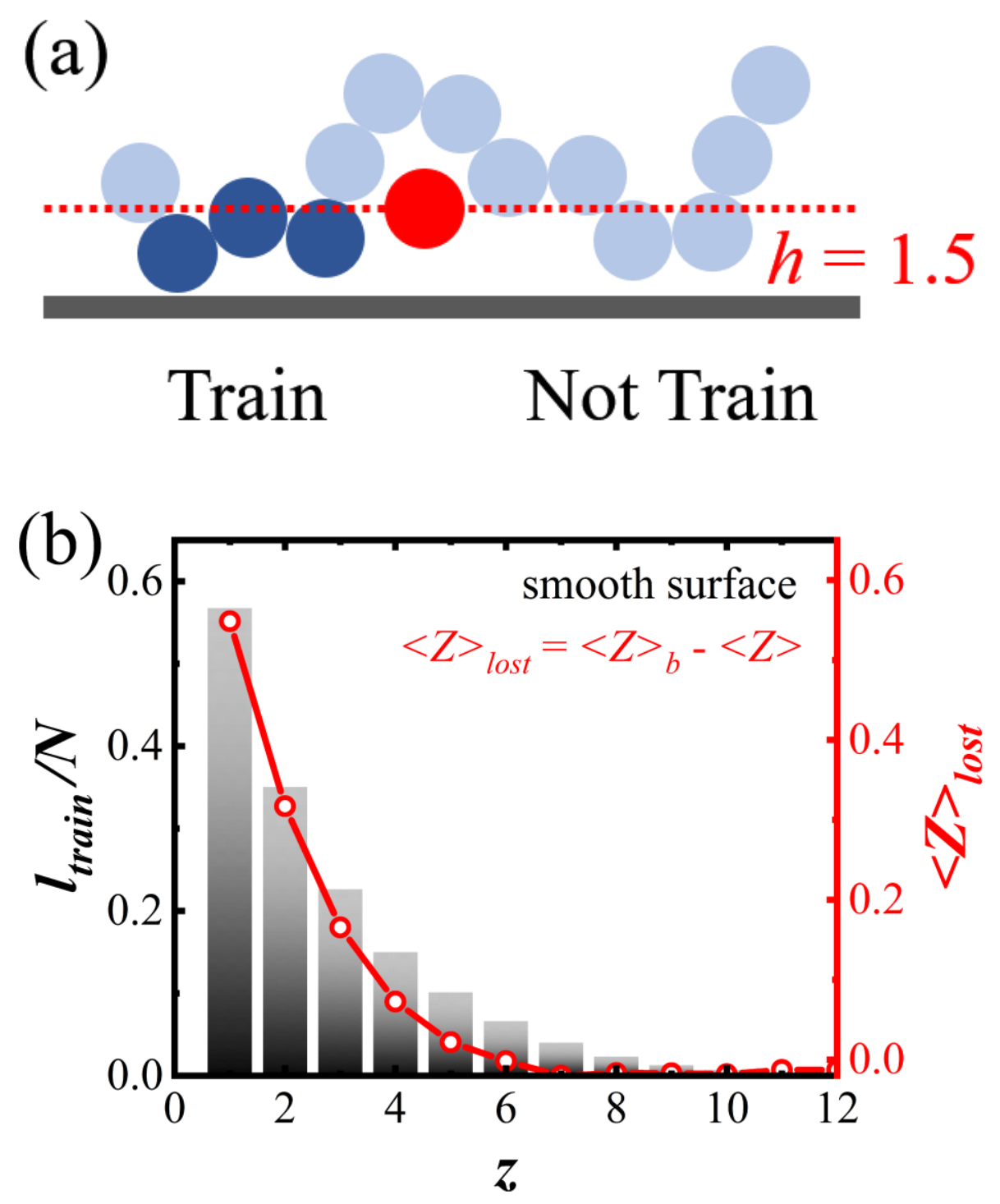

Figure S4: (a) Schematic representation of the definition of the train conformation, where the shallow blue circles indicate the monomers in the polymer chains, blue circles represent the train conformation, and red circles indicate the obstacle with a height of 1.5. (b) Length of train conformation per chain $l_{\text {train }}$ and the lost entanglements per chain $\langle Z\rangle_{\text {lost }}$ near the smooth surface as a function of $z$, where $\left\langle Z>_{\text {loss }}=\langle Z\rangle_{b}-\langle Z\rangle\right.$. 


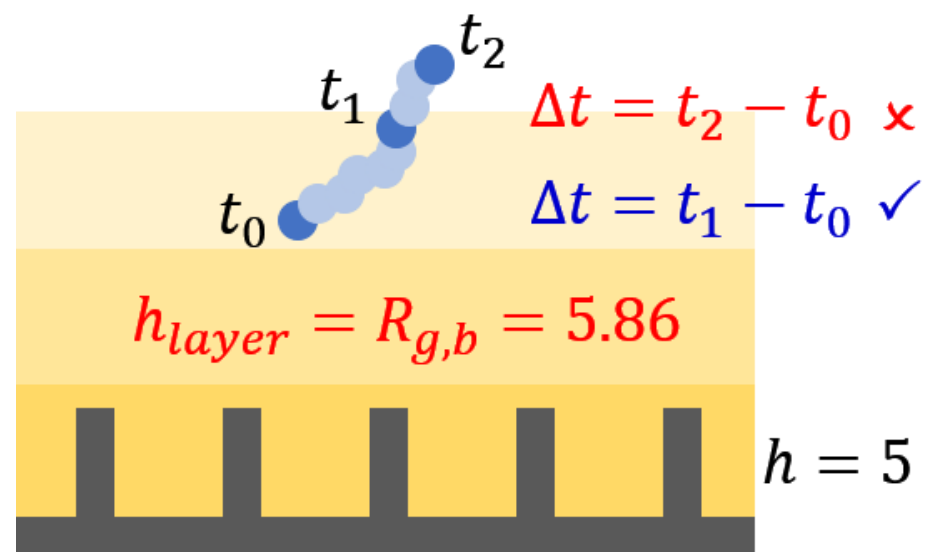

Figure S5: Schematic representation of the layered polymer film to investigate the polymer dynamics. The blue circles denote the monomers or the center of masses at different simulation times. During $t_{0}$ to $t_{1}$, the layer resolved mean squared displacement is calculated. 


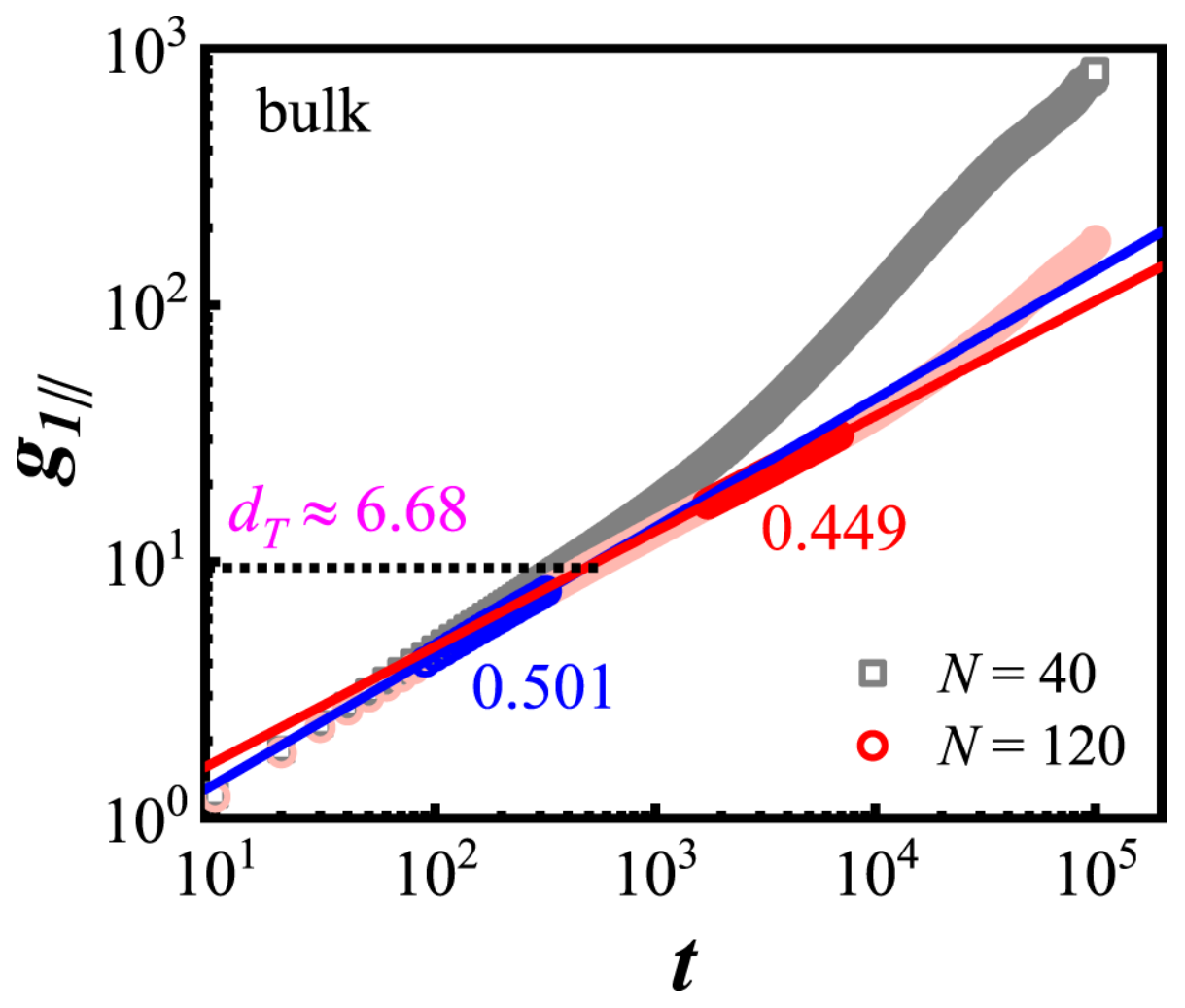

Figure S6: Parallel mean squared displacement of a central monomer $g_{I \|}$ as a function of the simulation time $t$ for a polymer bulk with two different chain lengths. The solid lines represent the best fitting of the data with the same color, and the dotted line denotes the corresponding value of $g_{1 \|}$ at the intersection $g_{I \|}{ }^{*}$ to calculate the diameter of the confined tube $d_{T}$. 


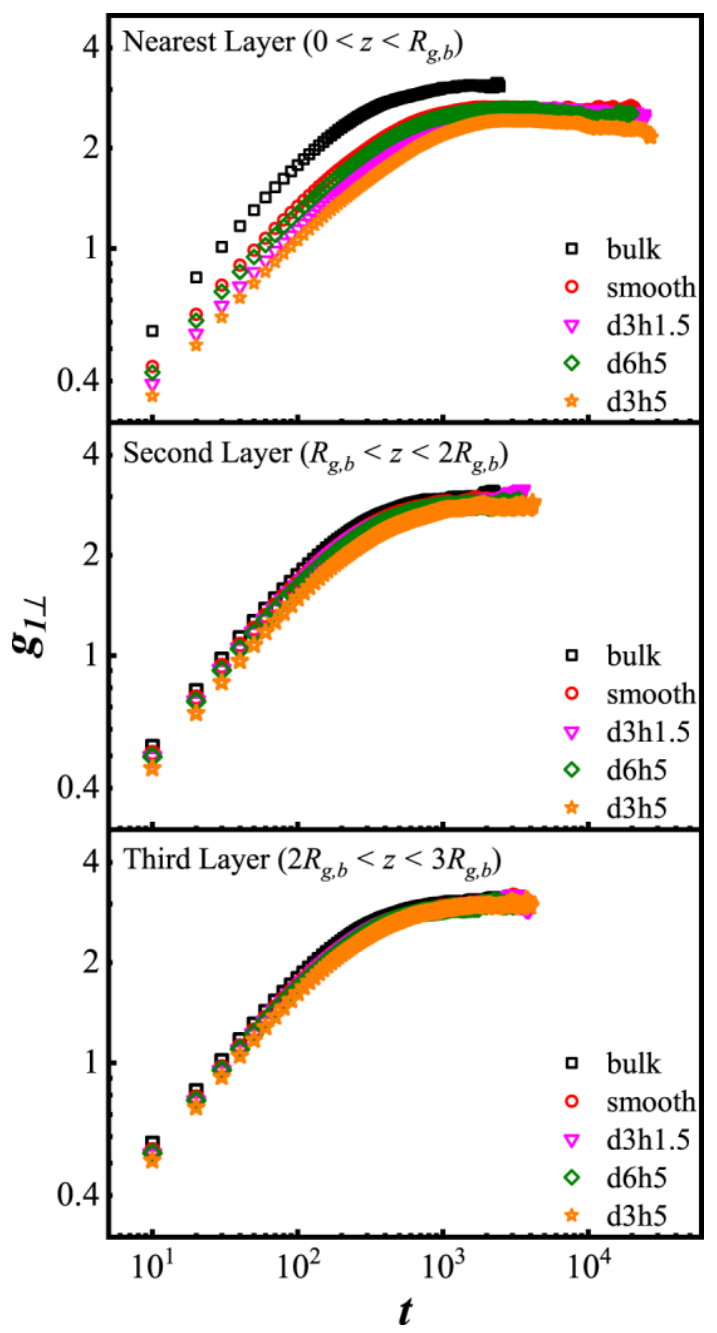

Figure S7: Layer resolved mean square displacement of a central monomer in the vertical direction $g_{1 \perp}$ as a function of the simulation time $t$. 

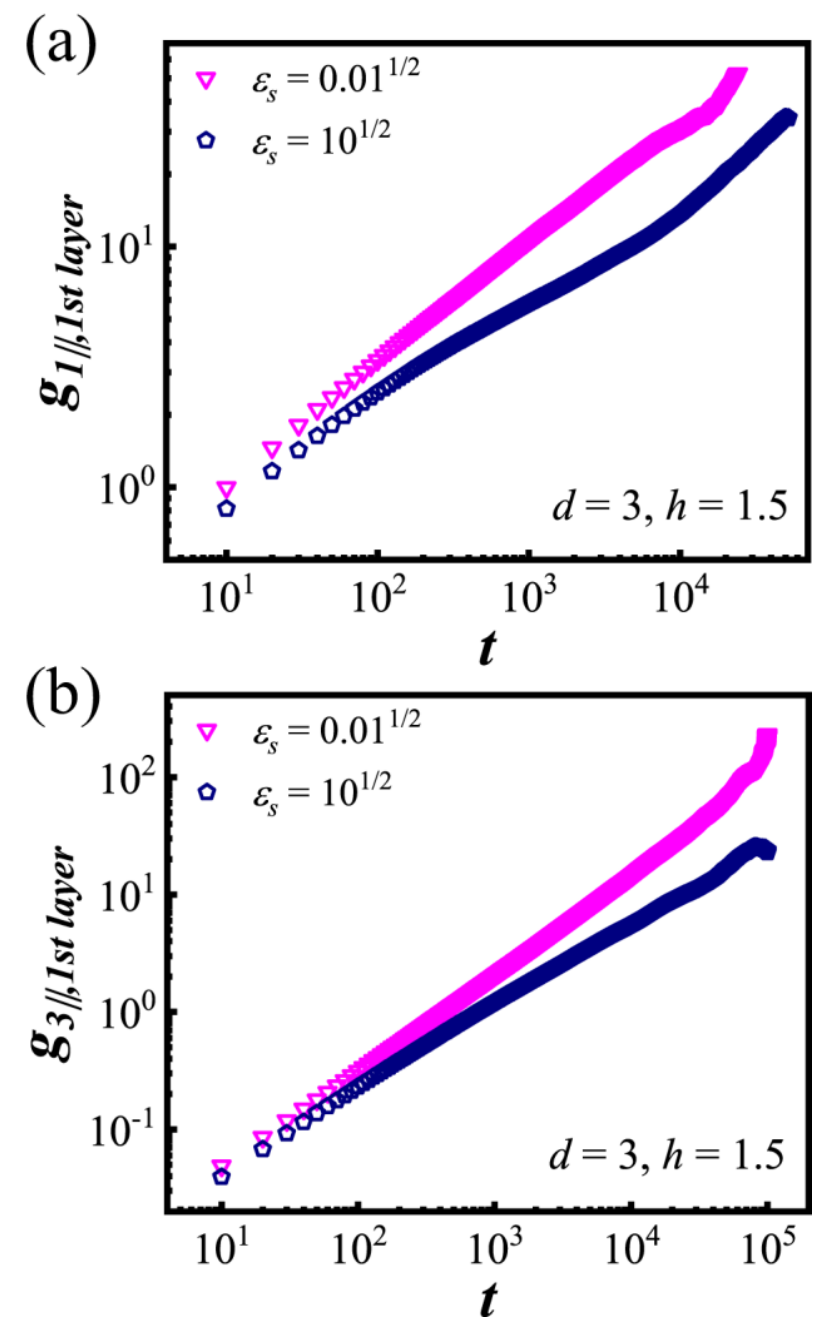

Figure S8: Parallel mean squared displacement of (a) a central monomer $g_{I||}$ and (b) the center of mass $g_{3||}$ at the nearest layer as a function of the simulation time $t$ for a rough surface with $d=3, h=1.5$ and different adsorption strengths $\varepsilon_{s}$. 